\title{
An Overview of the Anomaly-Induced Inflation
}

\author{
Ilya L. Shapiro ${ }^{\text {a }}$

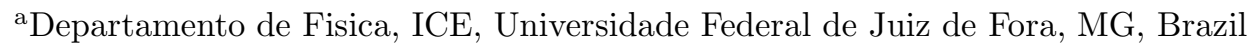

The anomaly-induced inflation (modified Starobinsky model) is based on the application of the effective quantum field theory approach to the Early Universe. We present a brief general review of this model with a special attention to the existing difficulties and unsolved problems.

The original version of the anomaly-induced inflation [ 1, 2, 3, 4] is the cosmological model which takes into account the vacuum quantum effects of the free, massless and conformally coupled to metric matter fields [ [5]. The quantum correction to the Einstein equation with cosmological constant

$R_{\mu \nu}-\frac{1}{2} R g_{\mu \nu}=8 \pi G<T_{\mu \nu}>-\Lambda$

produces a non-trivial effect because the anomalous trace of the stress tensor

$T=<T_{\mu}^{\mu}>=-\left(w C^{2}+b E+c \nabla^{2} R\right)$

is non-zero. If the matter fields are absent, there are the following equivalent ways to study the cosmological solution of (11): using the (0-0)component [1, 2] or via the anomaly-induced effective action [ 6, 7]. Indeed, the last option is completely equivalent to taking the trace of (11). The resulting equation has, for $k=0$ FRW metric, the following form (since the cases $k= \pm 1$ are quite similar [ 8 ] we will not consider them here):

$$
\begin{array}{r}
\frac{\dddot{a}}{a}+\frac{3 \ddot{a} \dddot{a}}{a^{2}}+\frac{\ddot{a}^{2}}{a^{2}}-\left(5+\frac{4 b}{c}\right) \frac{\ddot{a}^{2}}{a^{3}}- \\
-\frac{M_{P}^{2}}{8 \pi c}\left(\frac{\ddot{a}}{a}+\frac{\dot{a}^{2}}{a^{2}}-\frac{2 \Lambda}{3}\right)=0 .
\end{array}
$$

The equation above has a remarkable particular solution

$a(t)=a_{0} \cdot \exp (H t)$ where (motivated by the recent supernova data [ 9], we consider only positive cosmological constant in the low-energy regime)

$H=\frac{M_{P}}{\sqrt{-32 \pi b}}\left(1 \pm \sqrt{1+\frac{64 \pi b}{3} \frac{\Lambda}{M_{P}^{2}}}\right)^{1 / 2}$.

As far as $\Lambda \ll M_{P}^{2}$, we meet two very different solutions

$H_{c}=\sqrt{\frac{\Lambda}{3}} \quad$ and $\quad H_{S}=\frac{M_{P}}{\sqrt{-16 \pi b}}$.

The first solution is exactly the classical one, which one meets in the theory without quantum corrections, while the second one $H_{S}$ is the inflationary solution of Starobinsky.

The phase portrait of the theory may look very different depending on the sign of the coefficient $c$ [8]. The inflationary solution is stable for a positive $c$ and is unstable in the case $c<0$. In the last case there are several stable points (attractors), one of which corresponds to the FRW evolution. The original Starobinsky model deals only with the unstable solution. In this case one has to choose the initial conditions in a very special way. First of all, they must be very close to the exact exponential solution (5), such that the inflation lasts long enough. Moreover the choice of the initial condition has to provide that, after the inflationary phase ends, the Universe will approach the attractor corresponding to the FRW solution, and not to the other (physically unacceptable) attractor. All the matter content of the Universe is 
created after the inflation ends through the decay of the massive degree of freedom induced by anomaly [2, 3].

The Starobinsky model looks appealing, in particular because it is based on the quantum field theory results and one does not need to introduce a special inflaton field. At the same time, this theory is somehow more difficult for investigation than the inflaton-based models, moreover it requires at least the same amount of the fine-tuning for initial conditions as many inflaton models do. The importance of the very precise fine tuning in the 3-dimensional space of initial data is due to the existence of the non-FRW attractors corresponding to the physically unacceptable behaviors [10]. These solutions resemble the unphysical run-away solutions in QED and their possibility raised a generally suspicious relation to the idea of using quantum vacuum effects and higher derivative terms to achieve inflation. In order to advocate the whole approach, let us remark that the higher derivative terms must be included into the gravitational action anyway, because otherwise the quantum theory is inconsistent (see, e.g. [ [5] 7]). Then there are only two ways to protect the theory from the undesirable effects of the higher derivatives. The first is what has been done in [2] - choose the theory with the unstable inflation $c<0$ and fine-tune the initial data. But there is a second way - to opt for a positive $c$ and stability of the exponential solution at the beginning of inflation. The stable inflation is very robust with respect to the choice of the initial data and consequently it does not meet a problem with the unphysical solutions of the "run-away" sort.

The main problem of the stable inflationary model is to understand how the inflation ends. If we stay within the original framework [1, 2], that is consider only massless conformal fields, the stable inflation will be eternal, obviously contradicting our experience. The modified Starobinsky model solves this problem using the effective quantum field theory approach. The central idea has been suggested in [11], in the framework of the physical interpretation of the stability condition formulated before in [10].

The coefficients $w, b$ and $c$ in (2) depend on the number of the matter fields

$$
\begin{gathered}
w=\frac{1}{360(4 \pi)^{2}}\left(3 N_{0}+18 N_{1 / 2}+36 N_{1}\right), \\
b=-\frac{1}{360(4 \pi)^{2}}\left(N_{0}+11 N_{1 / 2}+62 N_{1}\right), \\
c=\frac{1}{360(4 \pi)^{2}}\left(2 N_{0}+12 N_{1 / 2}-36 N_{1}\right) .
\end{gathered}
$$

Using these relations the condition of stable inflation $c>0$ can be cast into the form

$N_{1}<\frac{1}{3} N_{1 / 2}+\frac{1}{18} N_{0}$.

The stable inflation requires the theory to include many scalars and fermions, for a given number of vectors. In high energy physics vectors correspond to the fundamental interactions. Then, what is the physical interpretation of (8) ? It is easy to see that the last inequality is not satisfied for the MSM with $N_{1,1 / 2,0}=(12,24,4)$. However, it is satisfied for the MSSM with $N_{1,1 / 2,0}=$ $(12,32,104)$. The same must be true for any realistic supersymmetric model, because the supersymmetrization of the realistic model implies adding many fermion and scalar superpartners (sparticles) while the fundamental interactions are kept the same. Then the transition between stable and unstable inflation can be associated with the SUSY breaking. Let us remember that the SUSY breaking implies the special form of the mass spectrum, such that sparticles are very heavy compared to observable particles (otherwise we should already see SUSY in the accelerator experiments). Therefore it is clear that inflation becomes unstable when its energy scale becomes smaller than the masses of the most of the sparticles and these sparticles decouple. For definiteness, we shall associate the energy of inflation with the magnitude of the Hubble parameter $H$. Let us introduce the notation $M_{*}$ for the energy scale where the inequality (8) changes its sign to the opposite. The anomaly-induced inflation model assumes that the value of $H$ is decreasing with time $\dot{H}<0$ and that $H_{S}$ is just an initial value of $H$. The stable inflation becomes unstable at the instant $t_{f}$ which is defined as a solution of the equation $H\left(t_{f}\right)=M_{*}$.

Two recent relevant results of quantum field theory in curved space-time must be mentioned: 
i) The decoupling of the loops of massive fields in curved space-time really takes place [[12, 13]. Despite serious difficulties in observing decoupling for the cosmological and inverse Newton constants, the existing data are sufficient for the purposes of the anomaly-induced inflation. In particular, one can observe the smooth and monotone evolution of the coefficient $c$ with scale and also the change of its sign from positive to negative due to the decoupling of the sparticles [ [12].

ii) The derivation of the effective action for massive fields can be performed in an approximate but direct way using the conformal description of the massive fields [14]. This is achieved through the introduction of a new auxiliary scalar which enables one to establish a new conformal Noether identity in the classical theory. At the quantum level, one meets conformal anomaly which can be integrated similar to the massless case [14, 8]. The result of the whole procedure is compatible with the renormalization group and may be viewed as its physical interpretation in the specific inflationary framework. Let us notice that the general formulation of the renormalization group in curved space-time (see, e.g., [ 7]) is based on the Minimal Subtraction scheme of renormalization and hence does not allow a physical interpretation for the specific cases.

According to the method of [ 14, 8] the leading effect of the particle masses is that the Planck mass and the cosmological constant in the equation (4) and in the solution (6) must be replaced by the variable expressions

$$
\begin{aligned}
M_{P}^{2} & \rightarrow M_{P}^{2}(1-\tilde{f} \ln a), \\
\Lambda M_{P}^{2} & \rightarrow \Lambda M_{P}^{2}(1-\tilde{g} \ln a),
\end{aligned}
$$

where

$$
\begin{array}{r}
\tilde{f}=\frac{1}{3 \pi} \sum_{f} \frac{N_{f} m_{f}^{2}}{M_{P}^{2}}, \\
\tilde{g}=\frac{1}{4 \pi} \sum_{s} \frac{N_{s} m_{s}^{4}}{M_{P}^{2} \Lambda}-\frac{1}{\pi} \sum_{f} \frac{N_{f} m_{f}^{4}}{M_{P}^{2} \Lambda}
\end{array}
$$

and the sums are taken over all species of fermions and scalars with masses $m_{f}, m_{s}$ and multiplicities $N_{f}, N_{s}$. The numerical analysis shows that the unconstrained value of $\tilde{g}$ is difficult to inter- pret, hence for the sake of simplicity we shall suppose that the SUSY spectrum is constrained by the relation $\tilde{g} \cong 0$, and also assume a small value of $\Lambda$ in the inflationary period. Then the equation (9) admits the following approximate analytical solution for $\ln a(t)=\sigma(t)$ :

$\sigma(t)=H_{0} t-\frac{H_{0}^{2}}{4} \tilde{f} t^{2}$.

It is interesting that the numerical analysis confirms the parabolic dependence (13) with enormous precision $[8$.

The relation (13) can be used to evaluate the total number of the inflationary $e$-folds for different models of the SUSY breaking. The first option is MSSM with the value $M_{*} \sim 1 \mathrm{TeV}$. It is easy to see that in this case $\tilde{f} \sim\left(M_{*} / M_{P}\right)^{2}=$ $10^{-32}$ and therefore the total amount of the $e$ folds is $10^{32}$. The expected temperature of the Universe after the end of inflation can be evaluated from Einstein equation in a usual way $T \sim$ $\sqrt{M_{*} M_{P}}=10^{11} \mathrm{GeV}$, which is a standard estimate for the inflaton-based models. An opposite extreme is to suppose that the SUSY takes place only at a very high energies and is broken already at the GUT scale. Suppose $M_{*} \propto 10^{14} \mathrm{GeV}$. Then the total amount of $e$-folds is about $10^{10}$. The problem of this version of the SUSY breaking is that the expected temperature after the end of inflation is very high $T \sim 10^{16} \mathrm{GeV}$, such that inflation does not solve the monopole problem of GUT's. Hence, the anomaly-induced inflation really favors low-energy SUSY.

The problems of stability in the anomalyinduced inflation with respect to the perturbations of conformal factor and tensor degree of freedom will be given in the parallel presentations [ 8 . Let us consider some of the problems and potential difficulties of the anomaly-induced inflation.

I. The stability of the inflationary solution (15) at the initial stage of inflation is not absolutely safe, because the criterion $c>0$ concerns only the stability with respect to the perturbations of the conformal factor. We know that the metric has other degrees of freedom and the anomalyinduced effective action does not explain why the initial metric was homogeneous and isotropic. Even if the stability with the respect to the small 
tensor perturbations holds [ 8, it does not solve the problem completely, for the initial deviation from the homogeneous and isotropic metric could be very large. One can either suppose that these question may be answered within a more fundamental theory like strings or try to find the mechanism of the automatic isotropization of the metric in the framework of the semiclassical theory. The last possibility has been widely discussed in the literature starting from [15], and indeed there are real chances to solve the problem in the semiclassical effective framework.

II. Let us make a very important observation concerning the coefficient $c$ in (2). The value of $c$ can be modified by adding the $\int \sqrt{-g} R^{2}$-term to the classical action of vacuum. Furthermore, the different regularization schemes can produce, generally speaking, different results for this coefficient, and moreover the higher-loop effects in the theory with scalar fields produce the $\int \sqrt{-g} R^{2}$ type divergences, such that this term must be introduced from the very beginning at the classical level and its value must be fixed by the renormalization condition. Hence it is unclear whether this indefiniteness may affect the modified Starobinsky model which is essentially based on the sign flip of the coefficient $c$ due to the possible SUSY breaking. This problem has been recently analyzed in full details [ [16]. The conclusion relevant for us is that there is no ambiguity in the coefficient $c$ which can not be fixed by the renormalization condition. Hence, the realization of the program of the anomaly-induced inflation [ 11] depends on the special renormalization condition for the $\int \sqrt{-g} R^{2}$-term. This renormalization condition must be imposed in such a way that the classical $\int \sqrt{-g} R^{2}$-term, together with the regularization ambiguity, would be small compared to the quantum contribution, e.g. derived in the point-splitting regularization [17] (see also discussion in [18]). It is important to notice that this requirement is perfectly compatible with the renormalization group equation.

III. Let us finally comment on the relation between our model and the standard approach to inflation. The inflaton models were introduced [19] in the situation when all possibilities to obtain in- flation directly from particle physics did not look promising. From our point of view, these models represent a specific cosmological phenomenology (see, e.g. [ [20]) which, as any other phenomenology, should serve as a bridge between observational data and some fundamental theory. In the phenomenological setting the inflaton models have obvious advantages compared to the anomaly-induced inflation. First of all, they are much more developed and also definitely simpler to deal with. However, the theoretical interpretation of an inflaton model is not going to be easy. It is supposed that one can establish the form of the inflaton potential with some reasonable precision and then find a quantum field theory with a scalar field or fields, string theory etc which, after all quantum corrections are taken into account, produces exactly the same potential. The problem of establishing the form of potential may become more difficult, technically, for the growing amount of the observational data, but the idea to establish a theoretical counterpart may be not realistic at all if the inflation was not caused by a scalar field potential.

On the other hand, the anomaly-induced inflation is also, in part, phenomenological. At the present level of knowledge this model does not require a fine-tuning of initial data or other parameters, but it is improbable that this situation will persist when we start the analysis of the reheating and density perturbations. Their behavior will likely depend on the high energy particle spectrum, on the details of decoupling for the inverse Newton constant and also on the choice of the vacuum for these perturbations which can not be performed, at the present state of art, in a unique way. In part, the same concerns the choice of vacuum for the metric perturbations at the beginning of inflation [10].

One has to remember that the inflaton models are very useful as a reference point, because they always provide (maybe with more than one scalar field) the fit with experimental or observational data with any desirable precision. In this way one obtains better understanding of these data and this is extremely important for the development of more complicated theory-based models such as the anomaly-induced inflation. Per- 
haps, the best solution would be to present the anomaly-induced effective action in a standard metric-inflaton form. An attempt of this sort has been undertaken in [10]. It turned out that one can perform such reduction, but only if one is exclusively interested in the evolution of the conformal factor. Within this approximation it is possible to present the effective action induced by quantum effects of matter field as a a secondderivative metric-scalar theory which can be of course called a new inflaton model. However, if we are interested, e.g., in the metric perturbations, one has to introduce more complicated auxiliary tensor "inflatons" and the similarity with the usual inflationary models does not work.

All in all, despite the anomaly-induced inflation is not as developed as inflaton models, it represents an attractive alternative to them. In particular, it enables one to avoid the standard fine-tuning in the choice of the initial data, gives a good chance to have a natural graceful exit and also to control the amplitude of the gravitational perturbations [ 8 . Only further theoretical and phenomenological study of this and other models and their comparison with future experimental/observational data may eventually show which of the models is closer to realities of our Universe in the first instants of its history.

Acknowledgments: Author is grateful to $\mathrm{CNPq}$ for the fellowship and to FAPEMIG for the grant.

\section{REFERENCES}

1. M.V. Fischetti, J.B. Hartle and B.L. Hu, Phys. Rev. D20 (1979) 1757.

2. A.A. Starobinsky, Phys. Lett. 91B (1980) 99.

3. A. Vilenkin, Phys. Rev. D32 (1985) 2511.

4. P. Anderson, Phys. Rev. D28 (1983) 271; D29 (1984) 615; D29 (1986) 1567.

5. N.D. Birrell and P.C.W. Davies, Quantum fields in curved space (Cambridge Univ. Press, Cambridge, 1982).

6. I.L. Buchbinder, S.D. Odintsov and I.L. Shapiro, Phys. Lett. 162B (1985) 92.

7. I.L. Buchbinder, S.D. Odintsov and I.L. Shapiro, Effective Action in Quantum Gravity (IOP Publishing, Bristol, 1992).

8. A.M. Pelinson, I.L. Shapiro and F.I. Takakura, Nucl. Phys. 648B (2003) 417, see also the article in this Proceedings.

9. S. Perlmutter et al., Astrophys. J . 517 (1999) 565; A.G. Riess et al., Astrophys. J. 116 (1998) 1009.
10. J.C. Fabris, A.M. Pelinson, I.L. Shapiro, Grav. Cosmol. 6 (2000) 59; Nucl. Phys. B597 (2001) 539.

11. I.L. Shapiro, Int. Journ. Mod. Phys. 11D (2002) 1159

12. E.V. Gorbar and I.L. Shapiro, JHEP 02 (2003) 021.

13. E.V. Gorbar and I.L. Shapiro, JHEP 06 (2003) 004.

14. I.L. Shapiro, J. Solà, Phys. Lett. 530B (2002) 10.

15. Ya.B. Zeldovich, Letters to JETP 12 (1970) 433; V.N. Lukash and A.A. Starobinsky, JETP 66 (1974) 1515 ;

B.L. Hu and L. Parker, Phys. Rev. 17 (1978) 933; V.N. Lukash, I.D. Novikov, A.A. Starobinsky and Ya.B. Zeldovich, Nuovo Cim. 35B (1976) 293.

16. M. Asorey, E.V. Gorbar and I.L. Shapiro, Universality and Ambiguities in the Trace Anomaly, hep-th/0307187, Class. Quant. Grav., to appear.

17. S.M. Christensen, Phys. Rev. 17D (1978) 948.

18. S.W. Hawking, T. Hertog and H.S. Real, Phys. Rev. D63 (2001) 083504.

19. A. Linde, Phys. Lett. 108B (1981) 389; A. Albrecht and P.J. Steinhardt, Phys. Rev. Lett. 48 (1982) 1220.

20. E. Kolb, Cosmology and the origin of structures. Lectures at the $\mathrm{X}$ Brazilian School of Cosmology and Gravitation. Mangaratiba, August, 2002. 